\title{
Regio- and stereoselective anomeric esterification of glucopyranose 1,2-diols and a facile preparation of 2-O-acetylated glucopyranosyl trichloroacetimidates from the corresponding 1,2-diols
}

\author{
Jianjun Zhang, ${ }^{\mathrm{a}}$ Xiaomei Liang, ${ }^{\mathrm{a}}$ Daoquan Wang ${ }^{\mathrm{a}, *}$ and Fanzuo Kong ${ }^{\mathrm{b}}$ \\ ${ }^{a}$ Key Lab of Pesticide Chemistry and Application Technology, Department of Applied Chemistry, \\ China Agricultural University, Beijing 100094, China \\ ${ }^{\mathrm{b}}$ Research Center for Eco-Environmental Sciences, Academia Sinica, PO Box 2871, Beijing 100085, China
}

Received 6 December 2006; received in revised form 15 January 2007; accepted 16 January 2007

Available online 24 January 2007

\begin{abstract}
A highly regio- and stereoselective anomeric esterification of 3- $O$-allyl (or benzyl, or benzoyl)-4,6- $O$-isopropylidene- $\alpha$, $\beta$ D-glucopyranose with acetyl chloride, or allyl chloroformate, or ethyl chloroformate gave the corresponding 2-OH, 1- $\beta$-acetates or -carbonates in excellent yields. The 2-OH, 1- $\beta$-acetates were readily converted to the corresponding 2-O-acetylated glucopyranosyl trichloroacetimidates by reaction with trichloroacetonitrile via base promoted acetyl migration, while the 2-OH, 1- $\beta$-carbonates were good glycosyl acceptors for the synthesis of $(1 \rightarrow 2)$-linked oligosaccharides.
\end{abstract}

(C) 2007 Elsevier Ltd. All rights reserved.

Keywords: Esterification; Acetyl chloride; Allyl chloroformate; Ethyl chloroformate; Glucopyranose 1,2-diols

\section{Introduction}

Regioselective introduction of protecting group is of crucial importance in carbohydrate chemistry. ${ }^{1}$ Acyl groups, especially acetyl and benzoyl, are generally used as electron-withdrawing protecting groups to block hydroxyls, and also used as good neighboring participating groups at 2-position for anomeric stereocontrol in glycosylation reactions. Although some selective protection approaches such as the use of 1-(benzoyloxy)benzotriazole $(\mathrm{BzOBT}),{ }^{2-5}$ the dibutyltin oxide mediated selective monoprotection strategy, ${ }^{6,7}$ the phase-transfer method, ${ }^{8}$ the silver(I) oxide promoted acylation technique, ${ }^{9}$ the $\mathrm{Cu}(\mathrm{II})$-mediated acylation procedure, ${ }^{10}$ or the selective activation of hydroxyl groups through stannylene compounds, ${ }^{11}$ as well as enzyme methods ${ }^{12}$ are available, these methodologies are mostly focused

\footnotetext{
*Corresponding author. Tel./fax: +86 10 62732219; e-mail: wangdq@ cau.edu.cn
}

on the selective acylation of sugar 2,3- or 4,6-diols. Regioselective protection at the anomeric hydroxyl group is, however, a challenge for chemists. ${ }^{4,13}$

Previously, we have revealed that selective acylation of allyl $4,6-O$-isopropylidene- $\alpha$-D-mannopyranoside ${ }^{14}$ or allyl 4- $O$-benzoyl- $\alpha$-L-rhamnopyranoside ${ }^{15}$ with acetyl chloride or chloroacetyl chloride in pyridine gave highly selective 3-O-acylation products, and with this method, a series of complex rhamnose and mannose oligosaccharides were synthesized efficiently. ${ }^{14-18}$ As an extension to this method, we wish to report herewith the regioand stereoselective C-1-O-acylation of glucopyranose 1,2-diols and their use in the preparation of glucose trichloroacetimidate donors and oligosaccharides.

The synthesis of 4,6-O-isopropylidene-3- $O$-allyl- $(\mathbf{8})$, -3-O-benzyl- (9), and -3-O-benzoyl- $\alpha, \beta$-D-glucopyranose (10) is depicted in Scheme 1. Allylation, or benzylation, or benzoylation of 1,2:5,6-di- $O$-isopropylidene- $\alpha$-D-glucofuranose (1) gave compounds $2,{ }^{19}$ or 3 , or $4,{ }^{20}$ respectively. Subsequent hydrolysis was carried out in an aqueous solution of sulfuric acid (4\%) under heating at 


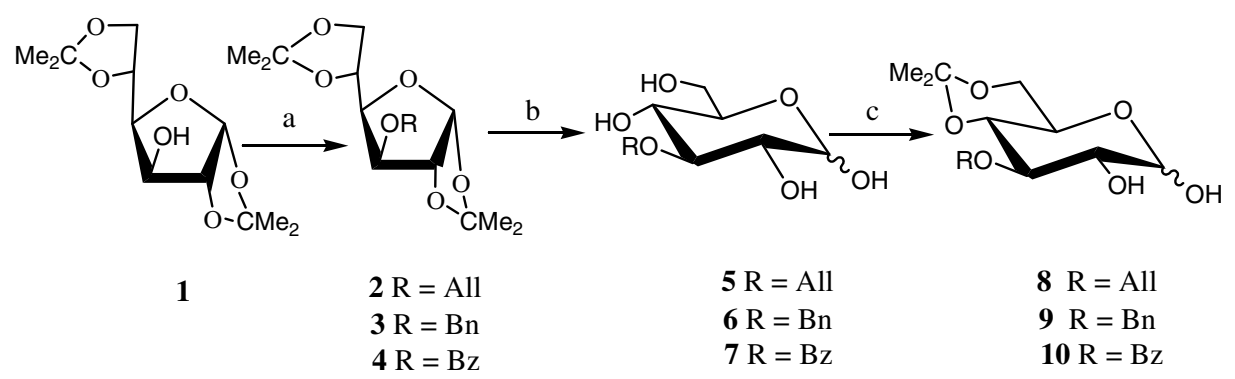

Scheme 1. Reagents and conditions: (a) AllBr, $\mathrm{NaH}, \mathrm{DMF}$ for 2; $\mathrm{BnBr}, \mathrm{NaH}$, DMF for 3; BzCl-pyridine for 4; (b) 4\% $\mathrm{H}_{2} \mathrm{SO}_{4}, \mathrm{reflux}$, 3-4 h; (c) TsOH, DMF, 1.05 equiv 2-methoxypropene (overall yield from 1: $76 \%, 81 \%$, and $75 \%$ for $\mathbf{8}$, 9, and 10, respectively).

reflux, and the reaction was accompanied by ring expansion. ${ }^{19}$ Selective 4,6-O-isopropylidenation of the resultant tetraols 5-7 with 2-methoxypropene in DMF in the presence of catalytic amounts of $\mathrm{TsOH}$ afforded the target diol compounds. The above three steps were processed in a consecutive manner without chromatographic separation of the intermediates, making a much simplified preparation of $\mathbf{8 - 1 0}$.

The 1,2-diols 8-10 were then employed for the selective acylation studies. Benzoylation of $\mathbf{8}$ with an equivalent amount of benzoyl chloride in pyridine at $-15^{\circ} \mathrm{C}$ led to a mixture of $1-O-\mathrm{Bz}, 2-O-\mathrm{Bz}$, and $1,2-\mathrm{di}-O-\mathrm{Bz}$ products, indicating that the benzoylation was not selective. This phenomenon was also observed in stereoselective benzoylation of D-glucosamine derivatives. ${ }^{4}$ When diols 8-10 were treated with allyl chloroformate, or ethyl chloroformate, or acetyl chloride in dichloromethane at room temperature in the presence of 4 equiv of pyridine, their C-1 hydroxyl group was selectively blocked, and 1-allyloxyformates 11, 14, and 17, or 1-ethyloxyformates 12,15 , and 18 , or 1-acetates 13, 16, and 19 , with predominant $\beta$ anomer were obtained, respectively, after separation by silica gel column chromatography (Table 1, entries $1-9)$. Low temperature ( -15 to $-10^{\circ} \mathrm{C}$ ), slow addition of the dichloromethane diluted acetyl chloride or chloroformates were necessary to ensure the high regioselectivity of the reaction. The regioselectivity was indicated clearly by the ${ }^{1} \mathrm{H}$ NMR spectra of the 1-esters, which exhibited characteristic downfield signals for H-1. Since the proton of the 1hydroxy group is more acidic than the 2-hydroxy group, the high regioselectivity was not surprising.

As for the high $\beta$ anomeric stereoselectivity, it was attributed to the kinetic stereoelectronic effect or 1,3diaxial repulsion, ${ }^{21}$ enabling the $\mathrm{C}-1-\mathrm{O}^{-}$to take the equatorial orientation. Because of the poor solubility of $\mathbf{1 0}$ in dichloromethane, more pyridine (10 equiv) was used, and the ratio of $\alpha$ anomer rose considerably (Table 1, entries 7-9). The configuration of the products was readily assigned from their ${ }^{1} \mathrm{H}$ NMR spectra as indicated in Table 1 , that is, the $\alpha$ anomers showed small coupling constants $\left(J_{1,2} 3.3-4.0 \mathrm{~Hz}\right)$, while the $\beta$ anomers showed large coupling constants $\left(J_{1,2} 7.9-8.2 \mathrm{~Hz}\right)$.
Trichloroacetimidate has widely been used as an excellent leaving group in glycosidic bond formation, ${ }^{21}$ and also as a temporary protective group for hydroxyl function. ${ }^{22-24}$ In our research, transformation of the 2$\mathrm{OH}$ of the obtained glucosyl acetates and carbonates to the corresponding 2-trichloroacetimidate was studied. It was found that carbonates $\mathbf{1 4}$ and $\mathbf{1 5}$ were readily converted to the corresponding 2-trichloroacetimidate derivatives by addition of trichloroacetonitrile in the presence of $\mathrm{K}_{2} \mathrm{CO}_{3}$ and a catalytic amount of DBU, affording 20 and 21 in good yields, and no $\alpha / \beta$ anomerization was observed (Scheme 2).

However, in the case of acetates $\mathbf{1 3}$ and 16, the reactions gave quite different results. Trichloroacetimidation of $\mathbf{1 3}$ and $\mathbf{1 6}$ with trichloroacetonitrile under the same conditions gave the corresponding 2-O-acetylated glucopyranosyl trichloroacetimidates $24(84 \%)$ and $25(79 \%)$, respectively. Compounds $\mathbf{2 4}$ and $\mathbf{2 5}$ showed identical NMR data with those of the authentic samples prepared from 8 and 9 via 1,2-di-O-acetylation, 1-O-deacetylation, and trichloroacetimidation. The facile migration of acyl groups in partially acylated sugars under mildly alkaline conditions is well known and has been the subject of numerous reports in the literature. ${ }^{25,26}$ Apparently, migration of the anomeric acetate and anomerization occurred during the process of trichloroacetimidation as outlined in Scheme 3. Similar 1,2-Osilyl group migration ${ }^{27,28}$ has been noticed by Schmidt and co-workers. Trichloroacetimidates $\mathbf{2 4}$ and $\mathbf{2 5}$ are very valuable glucopyranosyl donors as they contain orthogonal protective groups, being capable of application in the synthesis of complex branched oligosaccharides. It was also found that transformation of $\mathbf{8}$ to $\mathbf{2 4}$ via $\mathbf{1 3}$ (or 9 to $\mathbf{2 5}$ via 16) can be conveniently performed in a one-pot manner, leading to a $70 \%$ overall yield for $\mathbf{2 4}$ (or $64 \%$ for $\mathbf{2 5}$ ), and thus the preparation of $\mathbf{2 4}$ or $\mathbf{2 5}$ was greatly simplified.

Our attention turned then toward the formation of $(1 \rightarrow 2)$-linked disaccharides from the obtained glucose $2-\mathrm{OH}$ acetate or $2-\mathrm{OH}$ carbonate acceptors since $(1 \rightarrow 2)$ linked oligosaccharides are abundant in nature. ${ }^{29-31}$ It was found that TMSOTf catalyzed condensation of 1 -acetate 16 with 2,3,4,6-tetra- $O$-benzoyl- $\alpha$-D-manno- 
Table 1. Selective esterification reactions of the glucose 1,2-diols

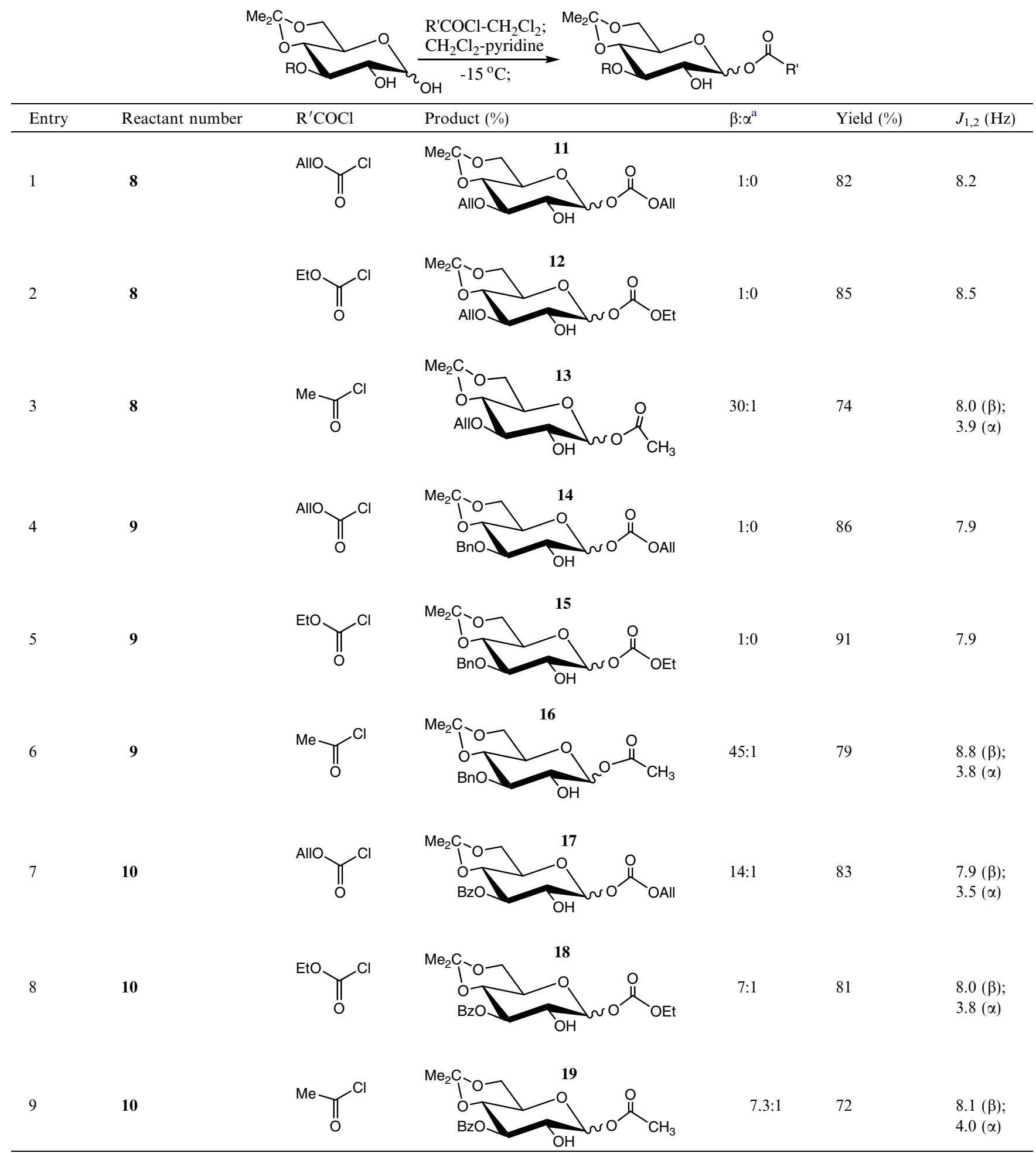

\footnotetext{
${ }^{\text {a }}$ The $\beta: \alpha$ ratio was estimated from the ${ }^{1} \mathrm{H}$ NMR spectra of the products.
}

pyranosyl trichloroacetimidate (26) was unsuccessful as a complex mixture was obtained. However, coupling reactions of carbonate $\mathbf{1 4}$ with trichloroacetimidates 26, 28, and $\mathbf{3 0}$ in the presence of catalytic TMSOTf under normal conditions ${ }^{32}$ produced disaccharides 27
(95\%), $29(88 \%)$, and 31 (91\%), respectively (Scheme 4). The anomeric center of the sugar residue at the reducing end of the obtained disaccharides kept its $\beta$ configuration as indicated in the NMR spectra of the disaccharides. Compared to the corresponding acetates, 

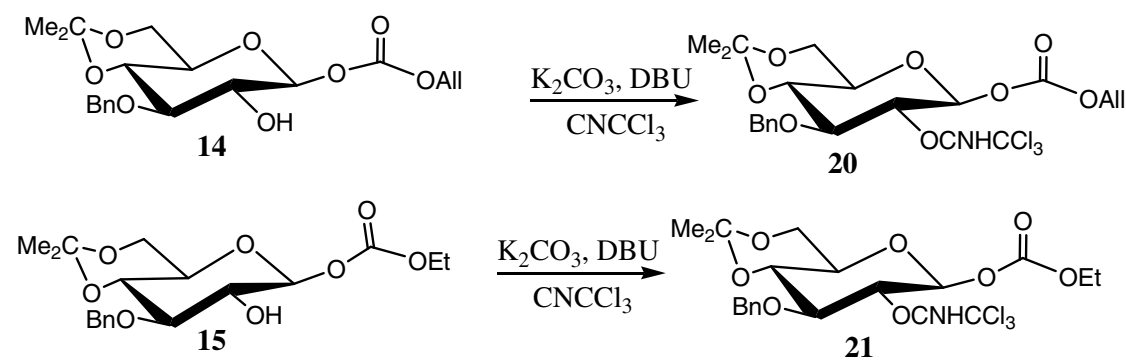

Scheme 2.

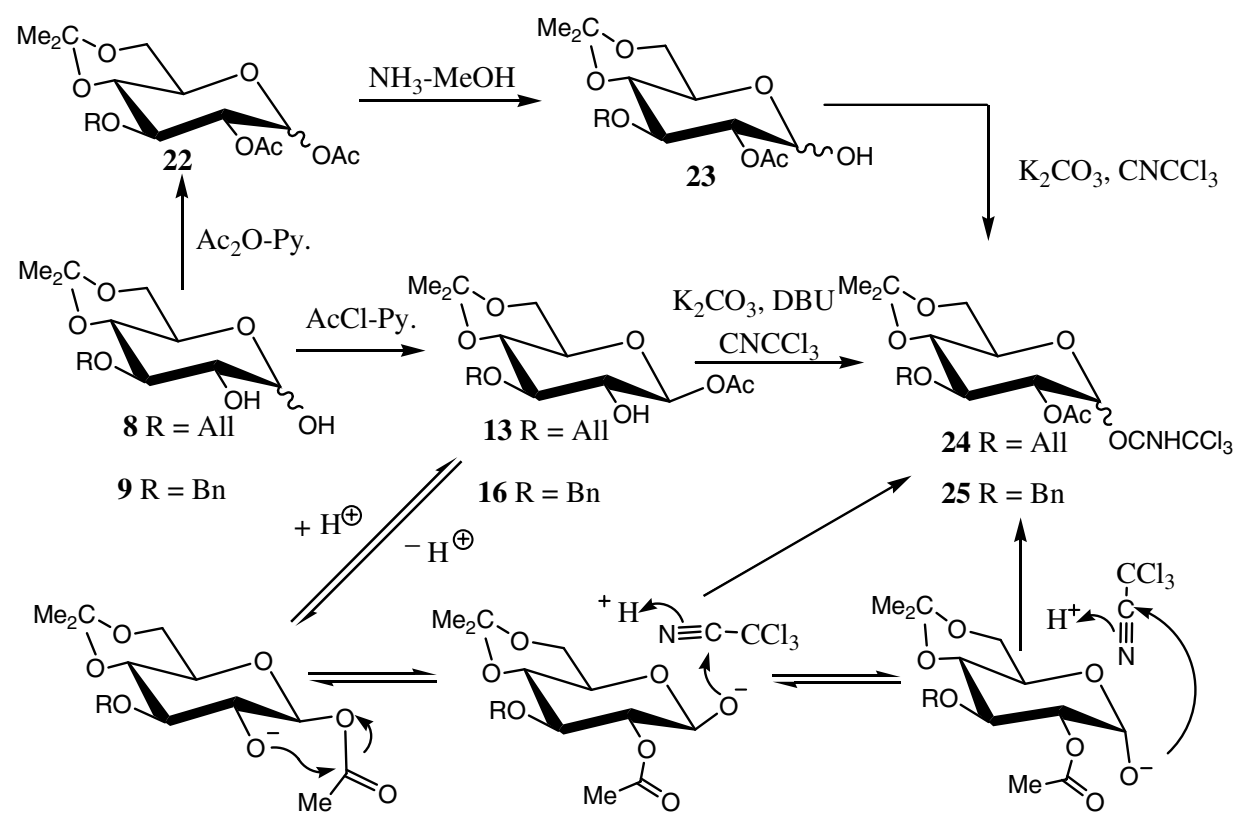

Scheme 3.

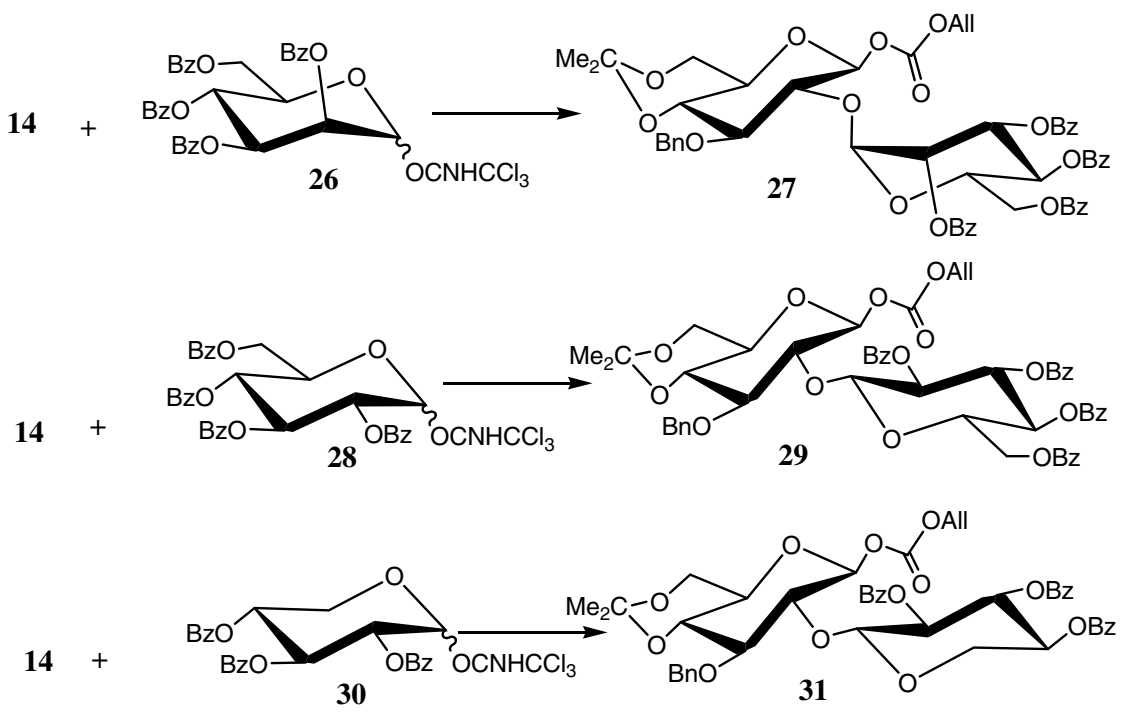

Scheme 4. Reagents and conditions: TMSOTf, $\mathrm{CH}_{2} \mathrm{Cl}_{2},-10{ }^{\circ} \mathrm{C}$ to $\mathrm{rt}$; $95 \%$ for $\mathbf{2 7}$ : $88 \%$ for $\mathbf{2 9}$, $91 \%$ for $\mathbf{3 1}$.

carbonates generally were much more stable presumably because of the resonance effect of the second oxygen.
In conclusion, we have successfully developed a technique for the highly regioselective and stereoselective 
esterification of 3-O-allyl (or benzyl, or benzoyl)-4,6- $O$ isopropylidene-D-glucopyranose with acetyl chloride, or allyl chloroformate, or ethyl chloroformate to afford the corresponding $2-\mathrm{OH}, 1-\beta$-acetates or -carbonates in good yields. With the aid of base promoted acetyl migration, an efficient preparation of the valuable 2-acetate 1-trichloroacetimidate donors from the corresponding glucopyranosyl 1,2-diols via the 1- $\beta$-acetate intermediates was achieved. The 2-OH, 1- $\beta$-carbonates were proved to be good acceptors in the coupling reactions for the preparation of $(1 \rightarrow 2)$-linked disaccharides.

\section{Experimental}

\subsection{General methods}

Optical rotations were determined with a Perkin-Elmer model 241-MC automatic polarimeter for soln in a 1$\mathrm{dm}$, jacketed cell. NMR spectra were recorded in deuterochloroform soln with a Bruker DPX300 spectrometer, using tetramethylsilane as an internal standard. Elemental analysis was performed on a Yanaco CHN Corder MF-3 automatic elemental analyzer. Thin-layer chromatography (TLC) was performed on Silica Gel HF with detection by charring with $30 \%$ (v/v) $\mathrm{H}_{2} \mathrm{SO}_{4}$ in $\mathrm{MeOH}$ or by UV detection. Column chromatography was conducted by elution of a column $(8 \times 100 \mathrm{~mm}, 16 \times$ $240 \mathrm{~mm}, 18 \times 300 \mathrm{~mm}, 35 \times 400 \mathrm{~mm}$ ) of Silica Gel (200-300 mesh) with EtOAc/petroleum ether (bp 60$90^{\circ} \mathrm{C}$ ) as the eluent. Analytical LC was performed with a Gilson HPLC consisting of a pump (model 306), stainless steel column packed with Silica Gel (Spherisorb $\mathrm{SiO}_{2}, 10 \times 300 \mathrm{~mm}$ or $4.6 \times 250 \mathrm{~mm}$ ), differential refractometer (132-RI Detector), and UV-vis detector (model 118). EtOAc-petroleum ether (bp $60-90{ }^{\circ} \mathrm{C}$ ) was used as the eluent at a flow rate of $1-4 \mathrm{~mL} / \mathrm{min}$. Solutions were concentrated at a temperature $<60{ }^{\circ} \mathrm{C}$ under diminished pressure.

\subsection{General procedure for the preparation of the gluco- pyranose 1,2-diols 8-10}

To a soln of 1 (7.80 g, $30 \mathrm{mmol})$ in DMF $(40 \mathrm{~mL})$ was added $95 \% \mathrm{NaH}(1.13 \mathrm{~g}, 45 \mathrm{mmol})$ in small portions at $0{ }^{\circ} \mathrm{C}$. The reaction mixture was stirred for $0.5 \mathrm{~h}$, at the end of which time allyl chloride or benzyl chloride $(35 \mathrm{mmol}$ ) was added dropwise. (For the synthesis of compound 10, compound 1 was benzoylated with 1.5 equiv of benzoyl chloride in pyridine, and the work-up procedure was according to the standard method.) After stirring for $2 \mathrm{~h}$ at room temperature, TLC (4:1 petroleum ether-EtOAc) indicated that the reaction was complete. The reaction mixture was quenched with $\mathrm{MeOH}(2 \mathrm{~mL})$, and diluted with $\mathrm{CH}_{2} \mathrm{Cl}_{2}(150 \mathrm{~mL})$, washed with $1 \mathrm{~N} \mathrm{HCl}$, water and satd aq $\mathrm{NaHCO}_{3}$.
The organic layer was concentrated, and the residue was dissolved in $4 \%$ aq $\mathrm{H}_{2} \mathrm{SO}_{4}(200 \mathrm{~mL})$ and then refluxed for $4 \mathrm{~h}$. The resulting soln was cooled down to room temperature and extracted 2 times with EtOAc. The organic phase was discarded and the aq phase was then stirred with $\mathrm{CaCO}_{3}(30 \mathrm{~g})$ for $2 \mathrm{~h}$. The reaction mixture was filtered and the residue was repeatedly washed with $\mathrm{MeOH}$. The combined filtrate and washings were evaporated under diminished pressure. The residual syrupy material was treated with a minimum vol of water and filtered in order to remove some insoluble material. The residue was washed with $\mathrm{MeOH}$ and the combined filtrate and washings were concentrated to give the 3-O-protected glucopyranose derivatives $\mathbf{5 - 7}$ as syrups. These syrups were dried under high vacuum for $4 \mathrm{~h}$ and then were taken in anhyd DMF $(80 \mathrm{~mL})$, $\mathrm{TsOH} \cdot \mathrm{H}_{2} \mathrm{O}(95 \mathrm{mg}, 0.5 \mathrm{mmol})$ and 2-methoxypropene $(3.6 \mathrm{~mL}, 36 \mathrm{mmol})$ were added successively under $\mathrm{N}_{2}$ atmosphere. The mixture was stirred at room temperature for $2 \mathrm{~h}$, and TLC (1:1 petroleum ether-EtOAc) indicated that the reaction was complete. $\mathrm{NaHCO}_{3}$ $(2.52 \mathrm{~g}, 30 \mathrm{mmol})$ was added to the reaction mixture, and the mixture was stirred for an additional $1 \mathrm{~h}$. After filtration, the mixture was concentrated under diminished pressure to give a residue, which was subjected to silica gel column chromatography (2:1 petroleum ether-EtOAc) to give the desired diol compounds.

2.2.1. 3-O-Allyl-4,6- $O$-isopropylidene- $\alpha, \beta$-D-glucopyranose (8). Yield $5.95 \mathrm{~g}$, overall yield from 1: 76\%, amorphous solid. $[\alpha]_{\mathrm{D}}^{22}+33\left(\right.$ c $\left.0.4, \mathrm{CHCl}_{3}\right) ;{ }^{1} \mathrm{H} \mathrm{NMR}\left(\mathrm{CDCl}_{3}\right.$, $300 \mathrm{MHz}): \delta 5.95-5.89\left(\mathrm{~m}, 1 \mathrm{H}, \mathrm{CH}_{2}=\mathrm{CH}-\mathrm{CH}_{2} \mathrm{O}\right), 5.26$ (d, $1 \mathrm{H}, J 3.1 \mathrm{~Hz}, \mathrm{H}-1, \alpha$-anomer), 5.32-5.15 (m, $2 \mathrm{H}$, $\left.\mathrm{CH}_{2}=\mathrm{CH}-\mathrm{CH}_{2} \mathrm{O}\right), 4.66(\mathrm{~d}, 1 \mathrm{H}, J 7.9 \mathrm{~Hz}, \mathrm{H}-1, \beta$-anomer), $4.40-4.33\left(\mathrm{~m}, 2 \mathrm{H}, \mathrm{CH}_{2}=\mathrm{CH}-\mathrm{CH}_{2} \mathrm{O}\right), 3.93-2.88$ $(\mathrm{m}, 6 \mathrm{H}), 1.49,1.41\left(2 \mathrm{~s}, 6 \mathrm{H}, \mathrm{Me}_{2} \mathrm{C}\right)$. Anal. Calcd for $\mathrm{C}_{12} \mathrm{H}_{20} \mathrm{O}_{6}$ : $\mathrm{C}, 55.37 ; \mathrm{H}, 7.74$. Found: $\mathrm{C}, 55.09 ; \mathrm{H}, 8.02$.

2.2.2. 3- $O$-Benzyl-4,6- $O$-isopropylidene- $\alpha, \beta$-D-glucopyranose (9). Yield $7.50 \mathrm{~g}$, overall yield from 1: 81\%, amorphous solid. $[\alpha]_{\mathrm{D}}^{22}+30\left(c 0.7, \mathrm{CHCl}_{3}\right) ;{ }^{1} \mathrm{H} \mathrm{NMR}\left(\mathrm{CDCl}_{3}\right.$, $300 \mathrm{MHz}): \delta 7.39-7.26(\mathrm{~m}, 5 \mathrm{H}, \mathrm{Ar}-H), 5.22(\mathrm{~d}, 1 \mathrm{H}, J$ $2.6 \mathrm{~Hz}, \mathrm{H}-1, \alpha$-anomer $), 4.92-4.69\left(\mathrm{~m}, 2 \mathrm{H}, \mathrm{CH}_{2} \mathrm{Ph}\right)$, $4.57\left(\mathrm{~d}, 1 \mathrm{H}, J_{1} 6.8 \mathrm{~Hz}, \mathrm{H}-1, \beta\right.$-anomer), 3.92-3.24 (m, $6 \mathrm{H}), 1.47,1.42\left(2 \mathrm{~s}, 6 \mathrm{H}, \mathrm{Me}_{2} \mathrm{C}, \beta\right.$-anomer $), 1.45,1.41$ (2s, $6 \mathrm{H}, \mathrm{Me}_{2} \mathrm{C}, \alpha$-anomer). Anal. Calcd for $\mathrm{C}_{16} \mathrm{H}_{22} \mathrm{O}_{6}$ : C, 61.92; H, 7.15. Found: C, 61.76; H, 7.30.

2.2.3. 3- $O$-Benzoyl-4,6- $O$-isopropylidene- $\alpha, \beta$-D-glucopyranose (10). Yield $7.30 \mathrm{~g}$, overall yield from 1: $75 \%$, foamy solid. $[\alpha]_{\mathrm{D}}^{22}+26\left(c 0.8, \mathrm{CHCl}_{3}\right) ;{ }^{1} \mathrm{H}$ NMR $\left(\mathrm{CDCl}_{3}\right.$, $300 \mathrm{MHz}): \delta 8.08-7.43(\mathrm{~m}, 5 \mathrm{H}, \mathrm{Bz}-H), 5.39$ (dd, $J$ 9.5, $9.7 \mathrm{~Hz}, \mathrm{H}-3, \alpha$-anomer), $5.32(\mathrm{~d}, 1 \mathrm{H}, J 3.7 \mathrm{~Hz}, \mathrm{H}-1, \alpha-$ anomer), 5.25 (dd, $J 9.5,9.7 \mathrm{~Hz}, \mathrm{H}-3, \beta$-anomer), 4.84 (d, $1 \mathrm{H}, J 7.6 \mathrm{~Hz}, \mathrm{H}-1, \beta$-anomer), $4.05-3.39(\mathrm{~m}, 5 \mathrm{H})$, $1.50,1.43\left(2 \mathrm{~s}, 6 \mathrm{H}, \mathrm{Me}_{2} \mathrm{C}, \beta\right.$-anomer), 1.49, 1.42 (2s, 
$6 \mathrm{H}, \mathrm{Me}_{2} \mathrm{C}, \alpha$-anomer). Anal. Calcd for $\mathrm{C}_{16} \mathrm{H}_{20} \mathrm{O}_{7}: \mathrm{C}$, 59.25; H, 6.22. Found: C, 59.03; H, 6.30.

\subsection{General procedure for the preparation of compounds 11-19}

1,2-Diols $(10.0 \mathrm{mmol})$ were dissolved in dry $\mathrm{CH}_{2} \mathrm{Cl}_{2}$ $(40 \mathrm{~mL})$ containing pyridine $(40 \mathrm{mmol}$ for 8 and $\mathbf{9}$; $100 \mathrm{mmol}$ for 10), then under $\mathrm{N}_{2}$ atmosphere and stirring, a soln of allyl chloroformate, or ethyl chloroformate, or acetyl chloride $(11 \mathrm{mmol})$ in anhyd $\mathrm{CH}_{2} \mathrm{Cl}_{2}$ $(10 \mathrm{~mL})$ was added dropwise within $30 \mathrm{~min}$ at -15 to $-20^{\circ} \mathrm{C}$. The reaction mixture was slowly warmed to room temperature and stirred for $2 \mathrm{~h}$, at the end of which time TLC (3:1 petroleum ether-EtOAc) indicated that the reaction was complete. The reaction mixture was diluted with $\mathrm{CH}_{2} \mathrm{Cl}_{2}(100 \mathrm{~mL})$, washed with $1 \mathrm{~N}$ $\mathrm{HCl}$, satd aq $\mathrm{NaHCO}_{3}$, and the organic layer was dried over $\mathrm{Na}_{2} \mathrm{SO}_{4}$. The soln was concentrated under diminished pressure, and the residue was purified by column chromatography on a silica gel column (5:1 petroleum ether-EtOAc). Yields and spectral data of 11-19 are as follows.

2.3.1. 3-O-Allyl-1-O-allyloxycarbonyl-4,6- $O$-isopropylidene- $\boldsymbol{\beta}$-D-glucopyranose (11). Yield $2.82 \mathrm{~g} \quad(82 \%)$, syrup. $[\alpha]_{\mathrm{D}}^{22}+33\left(c 0.5, \mathrm{CHCl}_{3}\right) ;{ }^{1} \mathrm{H}$ NMR $\left(\mathrm{CDCl}_{3}\right.$, $300 \mathrm{MHz}): \delta$ 5.93-5.78 (m, $\left.2 \mathrm{H}, 2 \mathrm{CH}_{2}=\mathrm{CH}-\mathrm{CH}_{2} \mathrm{O}\right)$, $5.38(\mathrm{~d}, \quad 1 \mathrm{H}, \quad J \quad 8.2 \mathrm{~Hz}, \mathrm{H}-1), \quad 5.34-5.09(\mathrm{~m}, \quad 4 \mathrm{H}$, $\left.2 \mathrm{CH}_{2}=\mathrm{CH}-\mathrm{CH}_{2} \mathrm{O}\right), \quad 4.63-4.56 \quad\left(\mathrm{~m}, 2 \mathrm{H}, \quad \mathrm{CH}_{2}=\mathrm{CH}-\right.$ $\left.\mathrm{CH}_{2} \mathrm{O}\right), 4.40-4.10\left(\mathrm{~m}, 2 \mathrm{H}, \mathrm{CH}_{2}=\mathrm{CH}-\mathrm{CH}_{2} \mathrm{O}\right), 3.88(\mathrm{dd}$, $1 \mathrm{H}, J$ 5.2, $10.5 \mathrm{~Hz}, \mathrm{H}-6 \mathrm{a}), 3.67(\mathrm{dd}, 1 \mathrm{H}, J 10.5$, $10.1 \mathrm{~Hz}, \mathrm{H}-6 \mathrm{~b}$ ), 3.61 (dd, 1H, $J$ 8.2, $8.8 \mathrm{~Hz}, \mathrm{H}-3$ ), 3.50 $(\mathrm{dd}, 1 \mathrm{H}, J$ 8.2, $8.8 \mathrm{~Hz}, \mathrm{H}-2), 3.39(\mathrm{dd}, 1 \mathrm{H}, J 8.8$, $8.7 \mathrm{~Hz}, \mathrm{H}-4), 3.37-3.20(\mathrm{~m}, 1 \mathrm{H}, \mathrm{H}-5), 3.12$ (br s, $1 \mathrm{H}$, $\mathrm{OH}), 1.42,1.34\left(2 \mathrm{~s}, 6 \mathrm{H}, \mathrm{Me}_{2} \mathrm{C}\right)$. Anal. Calcd for $\mathrm{C}_{16} \mathrm{H}_{24} \mathrm{O}_{8}$ : C, 55.81; H, 7.02. Found: C, 55.53; H, 6.88.

2.3.2. 3-O-Allyl-1- $O$-ethyloxycarbonyl-4,6- $O$-isopropyl-

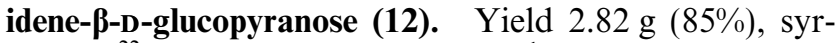
up. $\left.[\alpha]_{\mathrm{D}}^{22}+52(c) 0.9, \mathrm{CHCl}_{3}\right) ;{ }^{1} \mathrm{H} \quad \mathrm{NMR}\left(\mathrm{CDCl}_{3}\right.$, $300 \mathrm{MHz}): \delta \quad 6.00-5.87\left(\mathrm{~m}, 1 \mathrm{H}, \mathrm{CH}_{2}=\mathrm{CH}=\mathrm{CH}_{2} \mathrm{O}\right)$, $5.45(\mathrm{~d}, \quad 1 \mathrm{H}, J 8.5 \mathrm{~Hz}, \mathrm{H}-1), \quad 5.33-5.19(\mathrm{~m}, 2 \mathrm{H}$, $\left.\mathrm{CH}_{2}=\mathrm{CH}-\mathrm{CH}_{2} \mathrm{O}\right), \quad 4.42-4.35 \quad\left(\mathrm{~m}, \quad 1 \mathrm{H}, \quad 1 \mathrm{CH}_{2}=\mathrm{CH}-\right.$ $\mathrm{C} H \mathrm{HO}), \quad 4.28-4.17 \quad\left(\mathrm{~m}, \quad 3 \mathrm{H}, \quad 1 \mathrm{CH}_{2}=\mathrm{CH}-\mathrm{C} H \mathrm{HO}\right.$, $\left.\mathrm{CH}_{3} \mathrm{CH}_{2} \mathrm{O}\right), 3.94(\mathrm{dd}, 1 \mathrm{H}, J$ 5.3, $10.8 \mathrm{~Hz}, \mathrm{H}-6 \mathrm{a}), 3.75$ (dd, $1 \mathrm{H}, J 10.8,10.8 \mathrm{~Hz}, \mathrm{H}-6 \mathrm{~b}), 3.65(\mathrm{dd}, 1 \mathrm{H}, J 8.8$, $9.0 \mathrm{~Hz}, \mathrm{H}-3), 3.50(\mathrm{dd}, 1 \mathrm{H}, J 8.5,8.8 \mathrm{~Hz}, \mathrm{H}-2), 3.45$ (dd, $1 \mathrm{H}, J$ 9.0, $9.1 \mathrm{~Hz}, \mathrm{H}-4), 3.34-3.35(\mathrm{~m}, 1 \mathrm{H}, \mathrm{H}-5)$, 2.91 (br s, $1 \mathrm{H}, \mathrm{OH}), 1.49,1.41\left(2 \mathrm{~s}, 6 \mathrm{H}, \mathrm{Me}_{2} \mathrm{C}\right), 1.29$ (t, $3 \mathrm{H}, J 13.2 \mathrm{~Hz}, \mathrm{CH}_{3} \mathrm{CH}_{2} \mathrm{O}$ ). Anal. Calcd for $\mathrm{C}_{15} \mathrm{H}_{24} \mathrm{O}_{8}$ : C, 54.21; H, 7.28. Found: C, 54.52; H, 7.44.

2.3.3. 1-O-Acetyl-3- $O$-allyl-4,6- $O$-isopropylidene- $\alpha, \beta$-Dglucopyranose (13). Yield $2.23 \mathrm{~g}(74 \%)$, foamy solid. $[\alpha]_{\mathrm{D}}^{22}+49\left(c 1.0, \mathrm{CHCl}_{3}\right) ;{ }^{1} \mathrm{H} \mathrm{NMR}\left(\mathrm{CDCl}_{3}, 300 \mathrm{MHz}\right)$ : $\delta 6.24(\mathrm{~d}, 0.03 \mathrm{H}, J 3.90 \mathrm{~Hz}, \mathrm{H}-1, \alpha$-anomer), 6.02-5.80 $\left(\mathrm{m}, 1 \mathrm{H}, \mathrm{CH}_{2}=\mathrm{CH}-\mathrm{CH}_{2} \mathrm{O}, \alpha, \beta\right.$-anomer $), 5.58(\mathrm{~d}, 0.9 \mathrm{H}$,

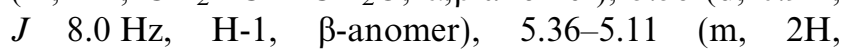
$\mathrm{CH}_{2}=\mathrm{CH}-\mathrm{CH}_{2} \mathrm{O}, \alpha, \beta$-anomer $), 4.42-4.14(\mathrm{~m}, 2 \mathrm{H}$, $\mathrm{CH}_{2}=\mathrm{CH}-\mathrm{CH}_{2} \mathrm{O}, \alpha, \beta$-anomer), $3.92(\mathrm{dd}, 0.9 \mathrm{H}, J$ 5.5, $10.7 \mathrm{~Hz}, \mathrm{H}-6 \mathrm{a}, \beta$-anomer), 3.76-3.38 (m, 5H, H-6a, $\alpha$ anomer; H-2-5, $\alpha, \beta$-anomer; H-6b, $\alpha, \beta$-anomer), 3.12 (br s, 1H, OH), 2.14 (s, 3H, $\beta-\mathrm{CH}_{3} \mathrm{CO}$ ), 2.09 (s, 3H, $\alpha$ $\left.\mathrm{CH}_{3} \mathrm{CO}\right), 1.49,1.41\left(2 \mathrm{~s}, 6 \mathrm{H}, \alpha, \beta-\mathrm{Me}_{2} \mathrm{C}\right)$. Anal. Calcd for $\mathrm{C}_{14} \mathrm{H}_{22} \mathrm{O}_{7}$ : C, 55.62; H, 7.33. Found: $\mathrm{C}, 55.35 ; \mathrm{H}$, 7.20 .

2.3.4. 1-O-Allyloxycarbonyl-3-O-benzyl-4,6- $O$-isopropylidene-p-D-glucopyranose (14). Yield $3.38 \mathrm{~g} \quad(86 \%)$, syrup. $[\alpha]_{\mathrm{D}}^{22}+41\left(c \quad 0.8, \mathrm{CHCl}_{3}\right) ;{ }^{1} \mathrm{H} \mathrm{NMR}\left(\mathrm{CDCl}_{3}\right.$, $300 \mathrm{MHz}): \delta$ 7.40-7.27 (m, 5H, Ar- H), 5.98-5.85 (m, $\left.1 \mathrm{H}, \mathrm{CH}_{2}=\mathrm{CH}-\mathrm{CH}_{2} \mathrm{O}\right), 5.45(\mathrm{~d}, 1 \mathrm{H}, J 7.9 \mathrm{~Hz}, \mathrm{H}-1)$, $5.40-5.25\left(\mathrm{~m}, 2 \mathrm{H}, \mathrm{CH}_{2}=\mathrm{CH}-\mathrm{CH}_{2} \mathrm{O}\right), 4.90(\mathrm{~d}, 1 \mathrm{H}, J$ $11.6 \mathrm{~Hz}, \mathrm{CHHPh}), 4.73(\mathrm{~d}, 1 \mathrm{H}, J 11.6 \mathrm{~Hz}, \mathrm{CHHPh})$, 4.68-4.64 (m, $\left.2 \mathrm{H}, \mathrm{CH}_{2}=\mathrm{CH}-\mathrm{CH}_{2} \mathrm{O}\right), 3.96(\mathrm{dd}, 1 \mathrm{H}, J$ 5.3, $10.7 \mathrm{~Hz}, \mathrm{H}-6 \mathrm{a}), 3.79-3.42$ (m, 4H, H-2, H-3, H-4, H-6b), 3.44-3.35 (m, 1H, H-5), 2.53 (br s, 1H, OH), 1.48, $1.42\left(2 \mathrm{~s}, 6 \mathrm{H}, \mathrm{Me}_{2} \mathrm{C}\right)$. Anal. Calcd for $\mathrm{C}_{20} \mathrm{H}_{26} \mathrm{O}_{8}$ : C, 60.90; H, 6.64. Found: C, 60.73; H, 6.78.

2.3.5. 3-O-Benzyl-1-O-ethyloxycarbonyl-4,6- $O$-isoprop-

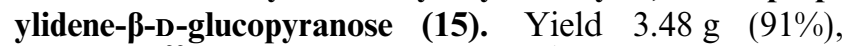
syrup. $\left.[\alpha]_{\mathrm{D}}^{22}+44(c) 1.2, \mathrm{CHCl}_{3}\right) ;{ }^{1} \mathrm{H} \mathrm{NMR}\left(\mathrm{CDCl}_{3}\right.$, $300 \mathrm{MHz}): \delta 7.36-7.25(\mathrm{~m}, 5 \mathrm{H}, \operatorname{Ar}-H), 5.44(\mathrm{~d}, 1 \mathrm{H}, J$ $7.9 \mathrm{~Hz}, \mathrm{H}-1), 4.93$ (d, 1H, J $11.7 \mathrm{~Hz}, \mathrm{CHHPh}), 4.73$ (d, $1 \mathrm{H}, J 11.7 \mathrm{~Hz}, \mathrm{CHHPh}), 4.26-4.19\left(\mathrm{~m}, 2 \mathrm{H}, \mathrm{CH}_{3} \mathrm{CH}_{2} \mathrm{O}\right)$, $3.94(\mathrm{dd}, 1 \mathrm{H}, J 5.2,10.6 \mathrm{~Hz}, \mathrm{H}-6 \mathrm{a}), 3.78-3.50(\mathrm{~m}, 4 \mathrm{H}$, H-2, H-3, H-4, H-6b), 3.43-3.35 (m, 1H, H-5), 2.61 (d, $1 \mathrm{H}, J 3.0 \mathrm{~Hz}, \mathrm{OH}), 1.47,1.42\left(2 \mathrm{~s}, 6 \mathrm{H}, \mathrm{Me}_{2} \mathrm{C}\right), 1.31(\mathrm{t}$, $3 \mathrm{H}, J 7.2 \mathrm{~Hz}, \mathrm{CH}_{3} \mathrm{CH}_{2} \mathrm{O}$ ). Anal. Calcd for $\mathrm{C}_{19} \mathrm{H}_{26} \mathrm{O}_{8}$ : C, 59.68; H, 6.85. Found: C, 59.66; H, 6.57.

2.3.6. 1-O-Acetyl-3- $O$-benzyl-4,6- $O$-isopropylidene- $\alpha, \beta$-Dglucopyranose (16). Yield $2.78 \mathrm{~g} \quad(79 \%)$, syrup. $[\alpha]_{\mathrm{D}}^{22}+42\left(c 1.3, \mathrm{CHCl}_{3}\right) ;{ }^{1} \mathrm{H} \mathrm{NMR}\left(\mathrm{CDCl}_{3}, 300 \mathrm{MHz}\right)$ : $\delta$ 7.36-7.24 (m, 5H, Ar- $H, \alpha, \beta$-anomer $), 5.35(\mathrm{~d}$, $0.02 \mathrm{H}, J 3.8 \mathrm{~Hz}, \mathrm{H}-1, \alpha$-anomer $), 4.84(\mathrm{~d}, 0.97 \mathrm{H}, J$ $8.8 \mathrm{~Hz}, \mathrm{H}-1, \beta$-anomer), 4.85-4.58 (m, $2 \mathrm{H}, \mathrm{CH}_{2} \mathrm{Ph}$, $\alpha, \beta$-anomer), 4.21 (dd, 0.02H, J 5.8, $10.5 \mathrm{~Hz}, \mathrm{H}-6 \mathrm{a}, \alpha$ anomer), 3.95-3.55 (m, 6H, H-2-6, $\alpha, \beta$-anomer), 2.05 (s, $2.9 \mathrm{H}, \mathrm{CH}_{3} \mathrm{CO}, \beta$-anomer), $2.03\left(\mathrm{~s}, 0.1 \mathrm{H}, \mathrm{CH}_{3} \mathrm{CO}\right.$, $\alpha$-anomer), $1.49,1.43\left(2 \mathrm{~s}, 6 \mathrm{H}, M e_{2} \mathrm{C}, \alpha, \beta\right.$-anomer $)$. Anal. Calcd for $\mathrm{C}_{18} \mathrm{H}_{24} \mathrm{O}_{7}: \mathrm{C}, 61.35 ; \mathrm{H}, 6.86$. Found: C, 61.30; H, 7.19.

2.3.7. 1-O-Allyloxycarbonyl-3- $O$-benzoyl-4,6- $O$-isopropylidene- $\boldsymbol{\alpha}, \boldsymbol{\beta}$-D-glucopyranose (17). Yield $3.39 \mathrm{~g}(83 \%)$, foamy solid. For $\beta$ isomer: $[\alpha]_{\mathrm{D}}^{22}+29\left(c 1.1, \mathrm{CHCl}_{3}\right)$; ${ }^{1} \mathrm{H}$ NMR $\left(\mathrm{CDCl}_{3}, 300 \mathrm{MHz}\right): \delta 8.07-7.44(\mathrm{~m}, 5 \mathrm{H}, \mathrm{Bz}-$ $H), 6.00-5.87\left(\mathrm{~m}, 1 \mathrm{H}, \mathrm{CH}_{2}=\mathrm{CH}-\mathrm{CH}_{2} \mathrm{O}\right), 5.58(\mathrm{~d}, 1 \mathrm{H}$, $J 7.9 \mathrm{~Hz}, \mathrm{H}-1), 5.45-5.29\left(\mathrm{~m}, 2 \mathrm{H}, \mathrm{CH}_{2}=\mathrm{CH}-\mathrm{CH}_{2} \mathrm{O}\right)$, 
$5.27(\mathrm{dd}, 1 \mathrm{H}, \quad J 9.3,9.2 \mathrm{~Hz}) 4.70-,4.65(\mathrm{~m}, 2 \mathrm{H}$, $\left.\mathrm{CH}_{2}=\mathrm{CH}-\mathrm{CH} \mathrm{H}_{2} \mathrm{O}\right), 4.03(\mathrm{dd}, 1 \mathrm{H}, J$ 5.4, $10.8 \mathrm{~Hz}, \mathrm{H}-6 \mathrm{a})$, 3.94-3.77 (m, 3H, H-2, H-4, H-6), 3.59-3.51 (m, 1H, $\mathrm{H}-5$ ), 3.08 (br s, 1H, OH), 1.50, 1.37 (2s, 6H, Me 2 C). Anal. Calcd for $\mathrm{C}_{20} \mathrm{H}_{24} \mathrm{O}_{9}$ : C, 58.82; H, 5.92. Found: C, 59.03; H, 5.77.

2.3.8. 3-O-Benzoyl-1- $O$-ethyloxycarbonyl-4,6- $O$-isopropylidene- $\boldsymbol{\alpha}, \boldsymbol{\beta}$-D-glucopyranose (18). Yield $3.21 \mathrm{~g}(81 \%)$, foamy solid. For $\beta$ isomer: $[\alpha]_{\mathrm{D}}^{22}+33\left(c\right.$ 1.0, $\left.\mathrm{CHCl}_{3}\right)$; ${ }^{1} \mathrm{H}$ NMR $\left(\mathrm{CDCl}_{3}, 300 \mathrm{MHz}\right): \delta 8.08-7.44(\mathrm{~m}, 5 \mathrm{H}, \mathrm{Bz}-$ $\mathrm{H}), 5.58(\mathrm{~d}, 1 \mathrm{H}, J 8.0 \mathrm{~Hz}, \mathrm{H}-1), 5.26(\mathrm{dd}, 1 \mathrm{H}, J 9.2$, $9.3 \mathrm{~Hz}, \mathrm{H}-3), 4.30-4.23\left(\mathrm{~m}, 2 \mathrm{H}, \mathrm{CH}_{3} \mathrm{CH}_{2} \mathrm{O}\right), 4.04$ (dd, $1 \mathrm{H}, J$ 5.0, $10.5 \mathrm{~Hz}, \mathrm{H}-6 \mathrm{a}), 3.92-3.76$ (m, 3H, H-2, H-4, H-6b), 3.59-3.50 (m, 1H, H-5), 3.00 (d, 1H, J $4.3 \mathrm{~Hz}$, $\mathrm{OH}), 1.49,1.37$ (2s, 6H, Me $2 \mathrm{C}), 1.33(\mathrm{t}, 3 \mathrm{H}, J 9.4 \mathrm{~Hz}$, $\mathrm{CH}_{3} \mathrm{CH}_{2} \mathrm{O}$ ). Anal. Calcd for $\mathrm{C}_{19} \mathrm{H}_{24} \mathrm{O}_{9}$ : C, 57.57; H, 6.10. Found: C, 57.84; H, 6.02.

2.3.9. 1- $O$-Acetyl-3- $O$-benzoyl-4,6- $O$-isopropylidene- $\alpha, \beta-$ D-glucopyranose (19). Yield $2.64 \mathrm{~g}(72 \%)$, foamy solid. $[\alpha]_{\mathrm{D}}^{22}+36\left(c 1.1, \mathrm{CHCl}_{3}\right) ;{ }^{1} \mathrm{H} \mathrm{NMR}\left(\mathrm{CDCl}_{3}, 300 \mathrm{MHz}\right): \delta$ 8.09-7.43 (m, 5H, Bz-H, $\alpha, \beta$-anomer), 6.21 (d, 0.11H, $J$ $4.0 \mathrm{~Hz}, \mathrm{H}-1, \alpha$-anomer), 5.73 (d, 0.88H, J 8.1 Hz, H-1, $\beta$-anomer), 5.40 (dd, 0.11H, J 9.2, $9.3 \mathrm{~Hz}, \mathrm{H}-3$, $\alpha$-anomer), 5.26 (dd, 0.88H, J 9.2, $9.3 \mathrm{~Hz}, \mathrm{H}-3, \beta$-anomer), 4.36-3.50 (m, 5H, H-2-6, $\alpha, \beta$-anomer), 2.97 (br s, $1 \mathrm{H}$, $\mathrm{OH}), 2.20\left(\mathrm{~s}, 0.33 \mathrm{H}, \mathrm{CH}_{3} \mathrm{CO}, \alpha\right.$-anomer $), 215(\mathrm{~s}$, $2.64 \mathrm{H}, \mathrm{CH}_{3} \mathrm{CO}, \beta$-anomer), 1.49, 1.37 (2s, 6H, Me ${ }_{2} \mathrm{C}$, $\alpha, \beta$-anomer). Anal. Calcd for $\mathrm{C}_{18} \mathrm{H}_{22} \mathrm{O}_{8}$ : C, 59.01; $\mathrm{H}$, 6.05. Found: C, 59.29; H, 6.23.

2.4. General procedure for the preparation of compounds 20, 21, 24, and 25

Compound 14 , or 15 , or 13 , or $16(5 \mathrm{mmol})$ and $\mathrm{K}_{2} \mathrm{CO}_{3}$ $(2.0 \mathrm{~g})$ were dried under high vacuum for $4 \mathrm{~h}$ and then $\mathrm{CH}_{2} \mathrm{Cl}_{2}(20 \mathrm{~mL})$ was added to the mixture. Then under $\mathrm{N}_{2}$ atmosphere, $\mathrm{CCl}_{3} \mathrm{CN}(1.0 \mathrm{~mL}, 10 \mathrm{mmol})$ was added, the mixture was stirred for $2 \mathrm{~h}$, at the end of which time DBU $(135 \mu \mathrm{L}, 0.9 \mathrm{mmol})$ was added. The reaction mixture was stirred for $12 \mathrm{~h}$, and TLC (2:1 petroleum etherEtOAc) indicated that the reaction was complete. After filtration, the mixture was concentrated under diminished pressure to give a residue, purification of the crude product on a silica gel column with 5:1 petroleum etherEtOAc as the eluent furnished the desired compounds.

2.4.1. 3-O-Benzyl-1- $O$-allyloxycarbonyl-4,6- $O$-isopropylidene-2- $\boldsymbol{O}$-trichloroacetimidoyl- $\beta$-D-glucopyranose $\mathbf{( 2 0 )}$. Yield $2.39 \mathrm{~g}(89 \%)$, syrup. $[\alpha]_{\mathrm{D}}^{22}+19\left(c 0.5, \mathrm{CHCl}_{3}\right) ;{ }^{1} \mathrm{H}$ $\mathrm{NMR}\left(\mathrm{CDCl}_{3}, 300 \mathrm{MHz}\right): \delta 8.65\left(\mathrm{~s}, 1 \mathrm{H}, \mathrm{CNHCCl}_{3}\right)$, 7.31-7.23 (m, 5H, Ar- $H)$, 5.94-5.68 (m, $1 \mathrm{H}$, $\left.\mathrm{CH}_{2}=\mathrm{CH}-\mathrm{CH}_{2} \mathrm{O}\right), 5.71(\mathrm{~d}, 1 \mathrm{H}, J 8.0 \mathrm{~Hz}, \mathrm{H}-1), 5.41$ $(\mathrm{dd}, 1 \mathrm{H}, \quad J 8.08 .1 \mathrm{~Hz}, \mathrm{H}-2), 5.38-5.23$ (m, 2H, $\left.\mathrm{CH}_{2}=\mathrm{CH}-\mathrm{CH}_{2} \mathrm{O}\right), 4.83-4.71\left(\mathrm{~m}, 2 \mathrm{H}, \mathrm{CH}_{2} \mathrm{Ph}\right), 4.67-$ $4.55\left(\mathrm{~m}, 2 \mathrm{H}, \mathrm{CH}_{2}=\mathrm{CH}-\mathrm{CH}_{2} \mathrm{O}\right), 4.01-3.75(\mathrm{~m}, 4 \mathrm{H}, \mathrm{H}-$ 3, H-4, H-6), 3.51-3.43 (m, 1H, H-5), 1.50, 1.43 (2s, $\left.6 \mathrm{H}, \mathrm{Me}_{2} \mathrm{C}\right)$. Anal. Calcd for $\mathrm{C}_{22} \mathrm{H}_{26} \mathrm{Cl}_{3} \mathrm{NO}_{8}$ : C, 49.04; H, 4.86; N, 2.60. Found: C, 49.31; H, 5.02; N, 2.98.

\subsubsection{3-O-Benzyl-1- $O$-ethyloxycarbonyl-4,6- $O$-isopropyl-} idene-2- $\boldsymbol{O}$-trichloroacetimidoyl- $\boldsymbol{\beta}$-D-glucopyranose (21). Yield $2.45 \mathrm{~g}(93 \%)$, syrup. $[\alpha]_{\mathrm{D}}^{22}+27\left(c 0.5, \mathrm{CHCl}_{3}\right) ;{ }^{1} \mathrm{H}$ $\mathrm{NMR}\left(\mathrm{CDCl}_{3}, 300 \mathrm{MHz}\right): \delta 8.65\left(\mathrm{~s}, 1 \mathrm{H}, \mathrm{CNHCCl}_{3}\right)$, 7.32-7.24 (m, 5H, Ar-H), 5.72 (d, 1H, J 8.0, H-1), 5.41 (dd, 1H, J 8.0, 8.1 Hz, H-2), 4.83-4.71 (m, 2H, $\mathrm{CH}_{2} \mathrm{Ph}$ ), $4.21\left(\mathrm{q}, 2 \mathrm{H}, J 7.1 \mathrm{~Hz}, \mathrm{OCH}_{2} \mathrm{CH}_{3}\right), 4.01-3.75$ (m, 4H, H3, H-4, H-6), 3.51-3.45 (m, 1H, H-5), 1.49, 1.43 (2s, 6H, $\left.\mathrm{Me}_{2} \mathrm{C}\right), 1.26\left(\mathrm{t}, 3 \mathrm{H}, \mathrm{J} 7.1 \mathrm{~Hz}, \mathrm{OCH}_{2} \mathrm{CH}_{3}\right)$. Anal. Calcd for $\mathrm{C}_{21} \mathrm{H}_{26} \mathrm{Cl}_{3} \mathrm{NO}_{8}$ : C, 47.88; H, 4.97; N, 2.66. Found: C, 47.75; H, 4.71; N, 2.29.

2.4.3. 2-O-Acetyl-3- $O$-allyl-4,6- $O$-isopropylidene- $\alpha, \beta$-Dglucopyranosyl trichloroacetimidate (24). Yield $1.87 \mathrm{~g}$ $(84 \%)$, syrup. $[\alpha]_{\mathrm{D}}^{22}+20 \quad\left(c \quad 0.5, \mathrm{CHCl}_{3}\right) ;{ }^{1} \mathrm{H} \quad \mathrm{NMR}$ $\left(\mathrm{CDCl}_{3}, 300 \mathrm{MHz}\right): \delta 8.66\left(\mathrm{~s}, 1 \mathrm{H}, \mathrm{CNHCCl}_{3}, \beta\right.$-anomer), 8.60 (s, $1 \mathrm{H}, \mathrm{CNHCCl}_{3}, \alpha$-anomer), $6.45(\mathrm{~d}, 1 \mathrm{H}$, $J 3.9 \mathrm{~Hz}, \mathrm{H}-1, \alpha$-anomer), 5.83 (d, 1H, J 8.2 Hz, H-1, $\beta$-anomer), 5.93-5.76 (m, $1 \mathrm{H}, \mathrm{CH}_{2}=\mathrm{CH}-\mathrm{CH}_{2} \mathrm{O}, \alpha, \beta$ anomer), 5.29-5.12 (m, $2 \mathrm{H}, \mathrm{CH}_{2}=\mathrm{CH}-\mathrm{CH}_{2} \mathrm{O}, \alpha, \beta$-anomer), 5.21 (dd, $1 \mathrm{H}, J 8.1,8.2 \mathrm{~Hz}, \mathrm{H}-2, \beta$-anomer), 4.99 (dd, 1H, J 3.9, 8.2 Hz, H-2, $\alpha$-anomer), 4.31-3.43 (m, $5 \mathrm{H}, \mathrm{H}-3, \mathrm{H}-4, \mathrm{H}-5, \mathrm{H}-6), 2.05$ (s, 3H, CH $\mathrm{H}_{3} \mathrm{CO}, \alpha$-anomer), $2.04\left(\mathrm{~s}, 3 \mathrm{H}, \mathrm{CH}_{3} \mathrm{CO}, \beta\right.$-anomer), $1.52,1.43(2 \mathrm{~s}$, $6 \mathrm{H}, \mathrm{Me}_{2} \mathrm{C}, \alpha$-anomer $), 1.51,1.42\left(2 \mathrm{~s}, 6 \mathrm{H}, \mathrm{Me}_{2} \mathrm{C}, \beta\right.$-anomer). Anal. Calcd for $\mathrm{C}_{16} \mathrm{H}_{22} \mathrm{Cl}_{3} \mathrm{NO}_{7}$ : C, 43.02; H, 4. 96; N, 3.14. Found: C, 42.83; H, 4.70; N, 3.30.

2.4.4. 2-O-Acetyl-3- $O$-benzyl-4,6- $O$-isopropylidene- $\alpha, \beta-$ D-glucopyranosyl trichloroacetimidate (25). Yield $1.96 \mathrm{~g}(79 \%)$, syrup. $[\alpha]_{\mathrm{D}}^{22}+12\left(c 0.5, \mathrm{CHCl}_{3}\right) ;{ }^{1} \mathrm{H} \mathrm{NMR}$ $\left(\mathrm{CDCl}_{3}, 300 \mathrm{MHz}\right): \delta 8.65\left(\mathrm{~s}, 1 \mathrm{H}, \mathrm{CNHCCl}_{3}, \beta\right.$-anomer), 8.58 (s, $1 \mathrm{H}, \mathrm{CNHCCl}_{3}, \alpha$-anomer), 7.35-7.24 (m, 5H, Ar- $H, \alpha, \beta$-anomer), 6.46 (d, $1 \mathrm{H}, J 3.8 \mathrm{~Hz}, \mathrm{H}-1, \alpha-$ anomer), $5.83(\mathrm{~d}, 1 \mathrm{H}, J 7.9 \mathrm{~Hz}, \mathrm{H}-1, \beta$-anomer $), 5.24$ (dd, 1H, J 7.8, $7.9 \mathrm{~Hz}, \mathrm{H}-2, \beta$-anomer), $5.00(\mathrm{dd}, 1 \mathrm{H}$, $J$ 3.8, $7.9 \mathrm{~Hz}, \mathrm{H}-2$, $\alpha$-anomer $), 4.88-4.64$ (m, 2H, $\mathrm{CH}_{2} \mathrm{Ph}, \alpha, \beta$-anomer $), 4.02-3.46(\mathrm{~m}, 5 \mathrm{H}, \mathrm{H}-3, \mathrm{H}-4, \mathrm{H}-$ 5, H-6), 1.97 (s, 3H, $\mathrm{CH}_{3} \mathrm{CO}, \alpha$-anomer), 1.96 (s, 3H, $\mathrm{CH}_{3} \mathrm{CO}, \beta$-anomer $), 1.51,1.45\left(2 \mathrm{~s}, 6 \mathrm{H}, \mathrm{Me}_{2} \mathrm{C}\right.$, $\alpha$-anomer), $1.50,1.44\left(2 \mathrm{~s}, 6 \mathrm{H}, \mathrm{Me}_{2} \mathrm{C}, \beta\right.$-anomer $)$. Anal. Calcd for $\mathrm{C}_{20} \mathrm{H}_{24} \mathrm{Cl}_{3} \mathrm{NO}_{7}$ : C, 48.36; H, 4.87; N, 2.82. Found: C, 48.00; H, 4.59; N, 3.05.

2.5. General procedure for the coupling reaction: synthesis of compounds 27, 29, and 31

Compound 12 (120 mg, $0.30 \mathrm{mmol})$ and sugar trichloroacetimidates $\mathbf{2 6}$, or $\mathbf{2 8}$, or $\mathbf{3 0}(0.36 \mathrm{mmol})$ were dried together under high vacuum for $4 \mathrm{~h}$, then dissolved in anhyd $\mathrm{CH}_{2} \mathrm{Cl}_{2}(10 \mathrm{~mL})$. TMSOTf $(1.8 \mu \mathrm{L}, 0.010 \mathrm{mmol})$ 
was added at $-10{ }^{\circ} \mathrm{C}$ under $\mathrm{N}_{2}$ atmosphere. The reaction mixture was stirred for $2 \mathrm{~h}$, during which time the temperature was gradually warmed to ambient temperature. Then the mixture was neutralized with $\mathrm{Et}_{3} \mathrm{~N}$ and concentrated to dryness under diminished pressure and the residue was purified by flash chromatography (4:1 petroleum ether-EtOAc). The physical data of the obtained disaccharides 27, 29, and $\mathbf{3 1}$ are as follows.

\subsubsection{2,3,4,6-Tetra- $O$-benzoyl- $\alpha$-D-mannopyranosyl-} $(1 \rightarrow 2)$-1- $O$-allyloxycarbonyl-3- $O$-benzyl-4,6- $O$-isopropyl-

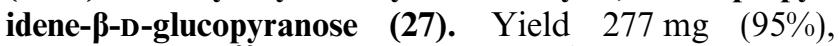
foamy solid. $[\alpha]_{\mathrm{D}}^{22}+14\left(c 1.2, \mathrm{CHCl}_{3}\right) ;{ }^{1} \mathrm{H} \mathrm{NMR}\left(\mathrm{CDCl}_{3}\right.$, $300 \mathrm{MHz}): \delta 8.10-7.17(\mathrm{~m}, 25 \mathrm{H}, 5 \mathrm{Ar}-H), 6.08(\mathrm{dd}, 1 \mathrm{H}, J$ $\left.10.2 \mathrm{~Hz}, \mathrm{H}-4^{\prime}\right), 5.86$ (dd, $\left.1 \mathrm{H}, J 3.3,10.2 \mathrm{~Hz}, \mathrm{H}-3^{\prime}\right), 5.95-$ $5.82\left(\mathrm{~m}, 1 \mathrm{H}, \mathrm{OCH}_{2} \mathrm{CH}=\mathrm{CH}_{2}\right), 5.67(\mathrm{~d}, 1 \mathrm{H}, J 8.0 \mathrm{~Hz}, \mathrm{H}-$ 1), $5.59\left(\mathrm{dd}, 1 \mathrm{H}, J 1.4,3.3 \mathrm{~Hz}, \mathrm{H}-2^{\prime}\right), 5.41(\mathrm{~d}, 1 \mathrm{H}, J$ $\left.1.4 \mathrm{~Hz}, \mathrm{H}-1^{\prime}\right), \quad 5.34-5.17\left(\mathrm{~m}, 2 \mathrm{H}, \quad \mathrm{OCH}_{2} \mathrm{CH}=\mathrm{CH}_{2}\right)$, 5.09-4.76 (m, 2H, $\left.\mathrm{CH}_{2} \mathrm{Ph}\right), 4.74-4.46(\mathrm{~m}, 3 \mathrm{H}), 4.30$ (dd, $\left.1 \mathrm{H}, J 2.1,12.5 \mathrm{~Hz}, \mathrm{H}-6^{\prime} \mathrm{a}\right), 4.04-3.73(\mathrm{~m}, 6 \mathrm{H})$, $3.15-3.44(\mathrm{~m}, 1 \mathrm{H}), 1.52,1.45\left(2 \mathrm{~s}, 6 \mathrm{H}, \mathrm{Me}_{2} \mathrm{C}\right) ;{ }^{13} \mathrm{C}$ NMR: $\delta 166.0,165.5,165.4,165.1,153.2,137.9,133.3$, $133.2,132.8,131.1,130.2,129.8,129.7,129.3,129.2$, $129.1,128.5,128.4,128.3,128.2,128.0,119.0,99.5$, 98.0, 96.9, 78.9, 77.2, 75.7, 74.8, 70.3, 69.8, 69.3, 68.9, 67.7, 66.6, 62.0, 29.0, 19.8. Anal. Calcd for $\mathrm{C}_{54} \mathrm{H}_{52} \mathrm{O}_{17}$ : C, 66.66; H, 5.39. Found: C, 66.84; H, 5.70.

2.5.2. $2,3,4,6$-Tetra- $O$-benzoyl- $\beta$-D-glucopyranosyl-(1 $\rightarrow 2)$ 1- $O$-allyloxycarbonyl-3- $O$-benzyl-4,6- $O$-isopropylidene- $\beta$ D-glucopyranose (29). Yield $257 \mathrm{mg}(88 \%)$, foamy solid. $[\alpha]_{\mathrm{D}}^{22}+8.0\left(\right.$ c 1.1, $\left.\mathrm{CHCl}_{3}\right) ;{ }^{1} \mathrm{H}$ NMR $\left(\mathrm{CDCl}_{3}\right.$, $300 \mathrm{MHz}): \delta 8.02-7.19(\mathrm{~m}, 25 \mathrm{H}, 5 \mathrm{Ar}-H), 5.85(\mathrm{dd}, 1 \mathrm{H}$, $\left.J 9.8 \mathrm{~Hz}, \mathrm{H}-4^{\prime}\right), 5.90-5.77\left(\mathrm{~m}, 1 \mathrm{H}, \mathrm{OCH}_{2} \mathrm{CH}=\mathrm{CH}_{2}\right), 5$. $68\left(\mathrm{dd}, 1 \mathrm{H}, J 9.8 \mathrm{~Hz}, \mathrm{H}-3^{\prime}\right), 5.67(\mathrm{~d}, 1 \mathrm{H}, J 8.9 \mathrm{~Hz}, \mathrm{H}-$ 1), $5.55\left(\mathrm{dd}, 1 \mathrm{H}, J 9.0,9.8 \mathrm{~Hz}, \mathrm{H}-2^{\prime}\right), 5.33-5.19(\mathrm{~m}$, $\left.2 \mathrm{H}, \mathrm{OCH}_{2} \mathrm{CH}=\mathrm{CH}_{2}\right), 522\left(\mathrm{~d}, 1 \mathrm{H}, J 9.0 \mathrm{~Hz}, \mathrm{H}-1^{\prime}\right)$, 4.65-4.32 (m, 6H), 4.08-3.84 (m, 3H), 3.72-3.53 (m, $3 \mathrm{H}), 3.36-3.21(\mathrm{~m}, 1 \mathrm{H}), 1.38,1.25\left(2 \mathrm{~s}, 6 \mathrm{H}, \mathrm{Me}_{2} \mathrm{C}\right) ;{ }^{13} \mathrm{C}$ NMR: $\delta 166.1,165.8,165.2,165.1,153.3,138.59$, $133.3,133.2,133.1,132.9,131.1,129.8,129.7,129.6$, $129.5,129.1,128.9,128.8,128.3,128.2,128.2,127.6$, $127.5,119.1,101.1,99.3,96.4,81.4,79.1,77.2,74.5$, $73.8,73.1,72.1,72.0,69.8,68.9,67.0,63.3,61.9,28.9$, 18.9. Anal. Calcd for $\mathrm{C}_{54} \mathrm{H}_{52} \mathrm{O}_{17}: \mathrm{C}, 66.66 ; \mathrm{H}, 5.39$. Found: C, 66.48; H, 5.24.

2.5.3. 2,3,4-Tri- $O$-benzoyl- $\beta$-D-xylopyranosyl-( $1 \rightarrow 2)-1-$ $O$-allyloxycarbonyl-3- $O$-benzyl-4,6- $O$-isopropylidene- $\beta$ D-glucopyranose (31). Yield $229 \mathrm{mg}$ (91\%), foamy, solid. $\left.[\alpha]_{\mathrm{D}}^{22}+22(c) 1.0, \mathrm{CHCl}_{3}\right) ;{ }^{1} \mathrm{H}$ NMR $\left(\mathrm{CDCl}_{3}\right.$, $300 \mathrm{MHz}): \delta 8.01-7.13(\mathrm{~m}, 20 \mathrm{H}, 4 \mathrm{Ar}-H), 5.93-5.80(\mathrm{~m}$, $\left.1 \mathrm{H}, \mathrm{OCH}_{2} \mathrm{CH}=\mathrm{CH}_{2}\right), 5.85\left(\mathrm{dd}, 1 \mathrm{H}, J 5.4 \mathrm{~Hz}, \mathrm{H}-3^{\prime}\right)$, $5.56(\mathrm{~d}, 1 \mathrm{H}, J 7.9 \mathrm{~Hz}, \mathrm{H}-1), 5.42(\mathrm{~d}, 1 \mathrm{H}, J 4.4 \mathrm{~Hz}, \mathrm{H}-$ $\left.1^{\prime}\right), 5.36-5.21\left(\mathrm{~m}, 3 \mathrm{H}, \mathrm{H}-2^{\prime}, \mathrm{OCH}_{2} \mathrm{CH}=\mathrm{CH}_{2}\right), 5.18(\mathrm{~m}$,
1H, H-4'), 4.75-4.41 (m, 5H), 4.00-3.69 (m, 6H), 3.44$3.35(\mathrm{~m}, 1 \mathrm{H}), 1.42,1.33\left(2 \mathrm{~s}, 6 \mathrm{H}, \mathrm{Me}_{2} \mathrm{C}\right)$. Anal. Calcd for $\mathrm{C}_{46} \mathrm{H}_{46} \mathrm{O}_{15}: \mathrm{C}, 65.86 ; \mathrm{H}, 5.53$. Found: $\mathrm{C}, 65.59 ; \mathrm{H}$, 5.44 .

\section{Acknowledgements}

This work was supported by The National Basic Research Program of China (2003CB114407) and the Research Foundation of China Agricultural University, Project No. 2006010.

\section{References}

1. Greene, T. W.; Wuts, P. G. M. Protective Groups in Organic Synthesis, 3rd ed.; John Wiley and Sons: New York, 1999.

2. Kim, S.; Chang, H.; Kim, W. J. J. Org. Chem. 1985, 50, 1751-1752.

3. Pelyvas, I. F.; Lindhorst, T. K.; Streicher, H.; Thiem, J. Synthesis 1991, 1015-1018.

4. Hung, S.; Thopate, S. R.; Wang, C. Carbohydr. Res. 2001, $330,177-182$.

5. Luo, S.; Kulkarni, S. S.; Chou, C.; Liao, W.; Hung, S. J. Org. Chem. 2006, 71, 1226-1229.

6. Roelens, S. J. Org. Chem. 1996, 61, 5257-5263.

7. Dong, L.; Roosenberg, J. M.; Miller, M. J. J. Am. Chem. Soc. 2002, 124, 15001-15005.

8. Garegg, P. J.; Kvarnstrom, I.; Niklasson, A.; Niklasson, G.; Svensson, S. C. T. J. Carbohydr. Chem. 1993, 12, 939953.

9. Wang, H.; She, J.; Zhang, L.; Ye, X. J. Org. Chem. 2004, $69,5774-5777$

10. Osborn, H.; Brome, V.; Harwood, L.; Suthers, W. Carbohydr. Res. 2001, 332, 157-166.

11. Grindley, T. B. Adv. Carbohydr. Chem. Biochem. 1998, 53, 17-26.

12. Schelhaas, M.; Waldmann, H. Angew. Chem., Int. Ed. 1996, 35, 2056.

13. Plusquellec, D.; Roullean, F.; Bertho, F.; Lefeuvre, M. Tetrahedron 1986, 42, 2457-2467.

14. Zhang, J.; Kong, F. Tetrahedron 2003, 59, 1429 1441.

15. Zhang, J.; Kong, F. Carbohydr. Res. 2003, 338, 17191725 .

16. Ma, Z.; Zhang, J.; Kong, F. Carbohydr. Res. 2004, 339, 43-49.

17. Ma, Z.; Zhang, J.; Kong, F. Carbohydr. Res. 2004, 339, 1761-1771.

18. Zhang, J.; Kong, F. J. Carbohydr. Chem. 2002, 21, 575585.

19. Bhattacharjee, A.; Datta, S.; Chattopadhyay, P.; Ghoshal, N.; Kundu, A. P.; Pal, A.; Mukhopadhyay, R.; Chowdhury, S.; Bhattacharjya, A.; Patra, A. Tetrahedron 2003, 59, 4623-4640.

20. Koen, A.; Jef, R.; Arthur Van, A.; Gerard, J.; Piet, H. J. Org. Chem. 1993, 58, 2977-2982.

21. Schmidt, R. R.; Kinzy, W. Adv. Carbohydr. Chem. Biochem. 1994, 50, 21-123.

22. Lassaletta, J. M.; Carlsson, K.; Garegg, P. J.; Schmidt, R. R. J. Org. Chem. 1996, 61, 6873-6880. 
23. Qiu, D.; Koganty, R. R. Tetrahedron Lett. 1997, 38, 961964.

24. Yu, B.; Yu, H.; Hui, Y.; Han, X. Synlett 1999, 753-755.

25. Haworth, W. N.; Hirst, E. L.; Teece, E. G. J. Chem. Soc. 1931, 2858-2859.

26. Bonner, W. A. J. Org. Chem. 1959, 24, 1388-1390.

27. Lassaletta, J. M.; Meichle, M.; Weiler, S.; Schmidt, R. R. J. Carbohydr. Chem. 1996, 15, 241-254.

28. Lassaletta, J. M.; Schmidt, R. R. Synlett 1995, 925-927.
29. Duefias-Chasco, M. T.; Rodriguez-Carvajal, M. A.; Mateo, P. T.; France-Rodriguez, G.; Espartero, J. L.; Irastorza-Iribas, A.; Gil-Serrano, A. M. Carbohydr. Res. 1997, 303, 453-458.

30. Wei, F.; Ma, S.-C.; Ma, L.-Y.; But, P. P.-H.; Lin, R.-C.; Khan, I. A. J. Nat. Prod. 2004, 67, 650-653.

31. Varki, A. Glycobiology 1993, 3, 97-130.

32. Schmidt, R. R. Angew. Chem., Int. Ed. Engl. 1986, 25, 212-235. 